\title{
Analysis of Microcapsule Resin Using the X-Ray Photoelectron Nitrogen 1s Spectral Method
}

\author{
Chiaki Inoue, Naoya Kobayashi, Mamoru Ishiguro, \\ and Kazunaka ENDO \\ Tsukuba Research Laboratory, Mitsubishi Paper Mills, Ltd., \\ 46 Wadai, Tsukuba, Ibaraki 300-42 Japan
}

(Received January 31, 1995)

\begin{abstract}
X-Ray photoelectron N 1s spectra have been used to determine which material is involved in the microcapsule; melamine or non-melamine (gelatin, polyurea and polyurethane). The linewidth of the $\mathrm{N} 1 \mathrm{~s}$ spectra of melamine-resin, $2.5 \mathrm{eV}$, is larger than that of non-melamine materials (polyurea $(2.1 \mathrm{eV})$ and gelatin $(1.9 \mathrm{eV})$ ). This depends on the nitrogen functional groups: The melamine involves two functional groups, amino and triazine-ring nitrogens, while polyurea and gelatin have amino groups. The $\mathrm{N} 1 \mathrm{~s}$ spectra of melamine-resin and polyurea were simulated by an $a b$ initio MO method using the melamine and 1,3-dimethyl urea molecules, respectively.

KEY WORDS Core XPS N 1s Spectra / Analysis / Ab Initio MO / Microcapsule Material / Melamine-Resin / Polyurea / Gelatin /
\end{abstract}

A microcapsule is used as a microscopic container which involves the dyes in recording materials such as carbonless duplicating, and heat-sensitive papers. This microcapsule is made of gelatin, melamine-resin, polyurea and polyurethane. We need a lot of time to analyze the capsule material chemically, since we must eliminate other substances involved in the capsule. For the melamine- and urea-resin, we have to examine each monomer after the resin was hydrolyzed by phosphoric aqueous solution. ${ }^{1}$ In the case of polyurea and polyurethane, the material must be identified from the ${ }^{1} \mathrm{H}$ NMR of the sample in polar organic solvents (dimethyl sulfoxide (DMSO), dimethyl acetoamide (DMA), and so on). ${ }^{2}$ In the present study, our aim is to analyze the capsule material using an X-ray photoelectron spectroscopy (XPS) method without the complicated chemical treatment.

As we know, there is no study on the analysis of the microcapsule material using the XPS method. We, here, noticed the X-ray photoelectron $\mathrm{N} 1 \mathrm{~s}$ spectra of the constituent element for the capsule, to determine which material is involved in the capsule; melamine or nonmelamine (gelatin, polyurea and so on). The difference between the melamine and nonmelamine was discussed with the linewidth of the $\mathrm{N}$ 1s spectra for the capsule.

\section{EXPERIMENTAL}

The X-ray photoelectron spectra were obtained on a PHI $5400 \mathrm{MC}$ spectrometer, using monochromatized $\mathrm{Al}-K_{\alpha}$ radiation. The spectrometer was operated at $400 \mathrm{~W}, 15 \mathrm{kV}$, and $26.7 \mathrm{~mA}$. The photon energy was $1486.6 \mathrm{eV}$. A pass energy of $35.75 \mathrm{eV}$ was employed for high-resolution scans in the $\mathrm{N} 1 \mathrm{~s}$ spectra region of $390-410 \mathrm{eV}$. The angle between the X-ray source and the analyzer was fixed at $45^{\circ}$. The spot size in the measurement was $3 \times 1 \mathrm{~mm}^{2}$. The use of dispersion compensation yielded an instrumental resolution of $0.5 \mathrm{eV}$ with the full width at half-maximum (FWHM) on the $\mathrm{Ag} 3 \mathrm{~d}$ line of silver. The reproductivity of the binding energy (BE) for $\mathrm{N}$ 1s spectra is within $\pm 0.1 \mathrm{eV}$. 
We used commercially-available gelatin (IK158; Nitta Gelatin, Ltd.), melamine, urea, and formalin. For gelatin, the sample is formed by cast-coating solution on aluminium plate. Two kinds of samples except gelatin were prepared by coating the microcapsule suspension on aluminium plate and produced by fixing the resin powder with a copper-metal-tape involving an acrylic-typed adhesive (3M copper tape). The microcapsule is generally produced in formation of shell material around oil drops in water. In this case, the oil drops are stably formed by addition of a surface active agent, the carboxylic sodium salt. We washed it out using a centrifuge, to purify the surface of the microcapsule. In the case of the capsule samples, we detected $\mathrm{Na}$ 1s spectra of the salts on the XPS measurements. We were able to neglect the effect in the present study.

\section{MOLECULAR ORBITAL CALCULATIONS}

We performed an ab initio molecular orbital (MO) calculation using the model molecules of the microcapsule materials, to compare the XPS N 1s binding energies with the theoretical core MO eigenvalues due to the Koopmans' theorem. We used melamine and 1,3-dimethyl urea instead of the melamine-resin and polyurea, respectively. The model molecules were calculated by the $a b$ initio restricted HartreeFock SCF MO method, using the HONDO7 program. ${ }^{3}$ For $\mathrm{C}, \mathrm{O}$, and $\mathrm{N}$ atoms, we used the $(4 \mathrm{~s} 2 \mathrm{p})$ basis sets and for $\mathrm{H}$ atoms we used the (2s) basis set reported by Huzinaga et al. ${ }^{4}$ and Dunning et al. ${ }^{5}$ for the geometry of the model molecules, we used the optimized values from the semi-empirical AM1 MO method. ${ }^{6}$

\section{RESULTS AND DISCUSSION}

It is interesting that we see the size of capsules from the photographs of microcapsules. The photographs of an scanning electron microscope (SEM) (Figure 1 (a) and (b)) showed microcapsules in the size range of a few to ten (a)

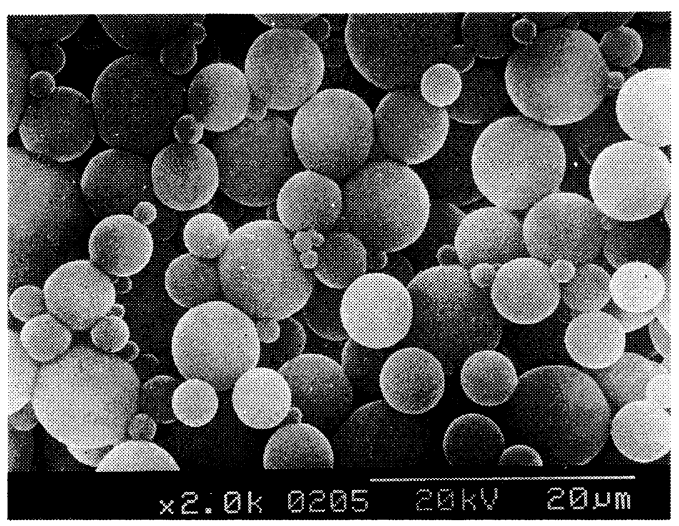

(b)

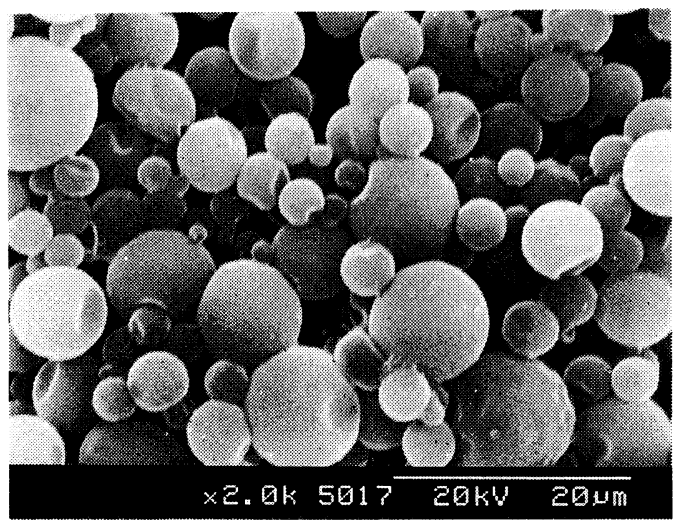

Figure 1. Photographs of microcapsules using a Scanning Electron Microscope. (a) melamine-resin shell; (b) polyurea shell.

$\mu \mathrm{m}$. We thought the material of the capsule shell can be analyzed with the core XPS N 1s spectra, since the analytical depth of the XPS is considered to be less than $5 \mathrm{~nm}$ and the thickness of the capsule shell approximates 10 to $100 \mathrm{~nm}$.

The most important point using XPS method is to study the bond-nature of the functional groups of the material elements. Let's consider the constituent groups of the capsule materials. For gelatin and polyurea, the nitrogen is a common-NH-bond; peptide (-NH-CO-) and urea (-NH-CO-NH-) bonds, respectively. In the case of melamine-resin, the material involves the two different bonding nitrogens. 


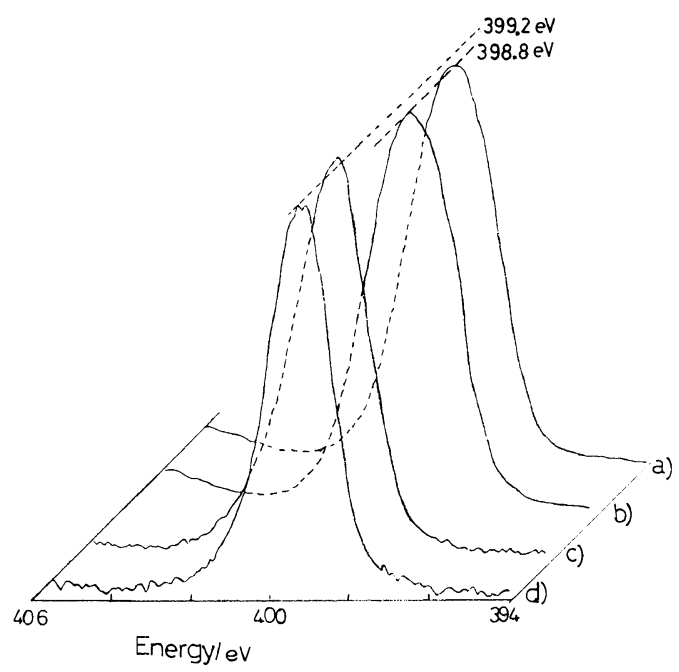

Figure 2. XPS N 1s spectra of microcapsule material. (a) melamine resin (powder); (b) melamine-resin (capsule); (c) polyurea (capsule); (d) gelatin (film).

Table I. Observed binding energy (BE) and full width of half-maximum (FWHM) of XPS N 1s spectra of materials for capsule shell

\begin{tabular}{|c|c|c|}
\hline \multirow{2}{*}{ Material } & $\mathrm{BE}$ & FWHW \\
\hline & $\mathrm{eV}$ & $\mathrm{eV}$ \\
\hline Melamine-resin (powder) & 398.8 & 2.5 \\
\hline Melamine-resin (capsule) & 398.8 & 2.5 \\
\hline Polyurea (capsule) & 399.3 & 2.1 \\
\hline Gelatin (film) & 399.2 & 1.9 \\
\hline
\end{tabular}

The one is $-\mathrm{NH}-\mathrm{CO}-$ bonds and the other the double $-\mathrm{C}=\mathrm{N}-$ bonds of 1,3,5-triazine ring. Then, we may be able to observe the single and double peaks of the XPS N 1s spectra for (gelatin and polyurea) and melamine-resin, respectively.

Figure 2 showed the single peaks of observed $\mathrm{N}$ 1s spectra for melamine-resin (powder and capsule), polyurea (capsule) and gelatin. We can see the differences of the $\mathrm{BE}$ and the FWHM of the spectra between melemine-resin and non-melamine (polyurea and gelatin) in Table I. This is due to the functional groups involved nitrogen.

For polyurea and melamine-resin, the simu-

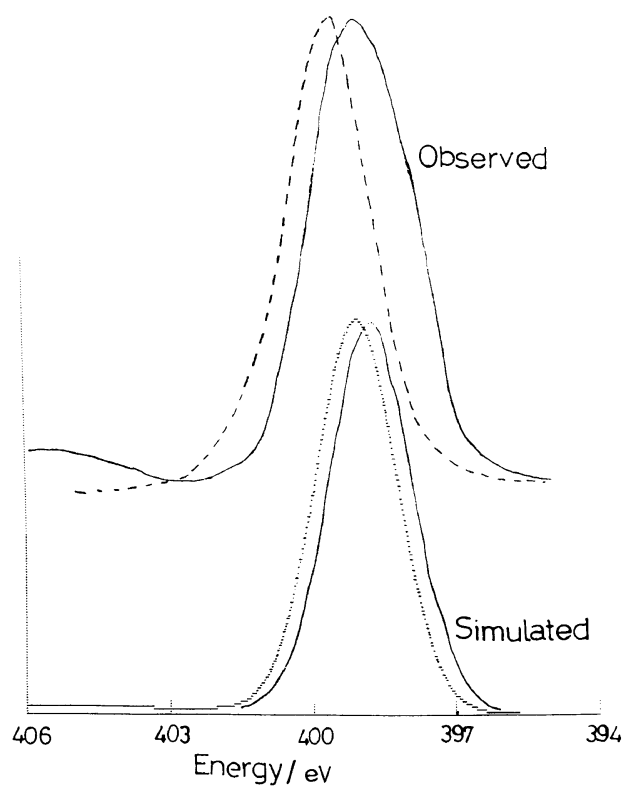

Figure 3. Simulated N 1s spectra of melamine-resin (solid line) and polyurea (dotted line) by an ab initio MO method using the model molecules (lower) and the observed spectra (upper).

Table II. Simulated BE and FWHM of XPS N 1s spectra of melamine-resin and polyurea using model molecules

\begin{tabular}{|c|c|c|c|c|c|}
\hline \multirow{3}{*}{ Material } & \multirow{3}{*}{$\frac{\begin{array}{c}\text { Koopmans' } \\
\text { value }^{\mathrm{a}}\end{array}}{\mathrm{eV}}$} & \multicolumn{2}{|c|}{ Calculated } & \multicolumn{2}{|c|}{ Observed } \\
\hline & & \multirow{2}{*}{$\frac{\mathrm{BE}}{\mathrm{eV}}$} & \multirow{2}{*}{$\frac{\text { FWHM }}{\mathrm{eV}}$} & \multirow{2}{*}{$\frac{\mathrm{BE}}{\mathrm{eV}}$} & \multirow{2}{*}{$\frac{\text { FWHM }}{\mathrm{eV}}$} \\
\hline & & & & & \\
\hline $\begin{array}{l}\text { Melamine- } \\
\text { resin }\end{array}$ & & 398.8 & 2.1 & 398.8 & 2.5 \\
\hline$-\mathrm{NH}$ & $424.0(399.0)$ & & & & \\
\hline$-\mathrm{N}=\mathrm{C}-$ & $423.6(398.6)$ & & & & \\
\hline Polyurea & $424.1(399.1)$ & 399.1 & 2.0 & 399.3 & 2.1 \\
\hline
\end{tabular}

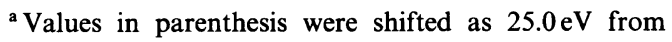
the Koopmans' theorem.

lated core $\mathrm{N}$ 1s spectra, as calculated by the MO method using the model molecules, seem to correspond to the XPS N 1s spectra of the capsule materials (as shown in Figure 3), although there were large differences in energy levels due to the Koopmans' theorem and work function and other energy effects. ${ }^{7}$ For this 
simulation, we used fixed energy shifts and fixed linewidths for calculated N 1s spectra. By trial and error, we found the energy shifted values as 25.0 for $\mathrm{N} 1 \mathrm{~s}$ spectra. The linewidth of each $\mathrm{MO}$ level for the $\mathrm{N} 1 \mathrm{~s}$ was evaluated $2.0 \mathrm{eV}$. The estimated BE and FWHM differences between melamine and 1,3-dimethyl urea agrees considerably well with the observed $\mathrm{N} 1 \mathrm{~s}$ spectra difference between melamine-resin and polyurea (as shown in Figure 3 and Table II). This indicates that we can obtain good assignments with the Koopmans' theorem due to the normal MO calculations of the ground state for $\mathrm{N}$ electrons using the ab initio HONDO program.

\section{REFERENCES}

1. The Japan Society for Analytical Chemistry, "Bunseki Kagakubinran," Maruzen, Ltd, Tokyo, 1981.

2. M. Sumi, Y. Chokki, Y. Nakai, M. Nakabayashi, and T. Kanzawa, Macromol. Chem., 78, 146 (1964).

3. M. S. Dupuis, J. D. Watts, H. G. Villar, and G. J. B. Hurst, HONDO, version 7; Scientific and Engineering Computations Dept. 48B, IBM Corp; New York, 12401; 1978.

4. S. Huzinaga, J. Andzelm, M. Klobukowski, E. Radzio-Andzelm, Y. Sakai, and H. Takewaki, "Gaussian Basis Sets for Molecular Calculations," Elsevior, Amsterdam, 1984.

5. T. H. Dunning, Jr. and P. J. Hay, in "Methods of Electronic Structure Theory," H. F. Schaefer, III, Ed., Plenum, New York, N. Y., 1977.

6. J. J. P. Stewart, J. Compt. Chem., 10, 289 (1988).

7. J. Delhalle, J. M. Andre, S. Delhalle, J. J. Pireaux, R. Caudano, and J. J. Verbist, J. Chem. Phys., 60, 595 (1974). 